\title{
Phonon-Assisted Population Inversion of a Single InGaAs/GaAs Quantum Dot by Pulsed Laser Excitation
}

\author{
J. H. Quilter, ${ }^{1}$ A. J. Brash, ${ }^{1}$ F. Liu, ${ }^{1,}{ }^{*}$ M. Glässl, ${ }^{2}$ A. M. Barth, ${ }^{2}$ V. M. Axt, ${ }^{2}$ A. J. Ramsay,${ }^{3}$ M. S. Skolnick,,${ }^{1}$ and A. M. Fox ${ }^{1}$ \\ ${ }^{1}$ Department of Physics and Astronomy, University of Sheffield, Sheffield S3 7RH, United Kingdom \\ ${ }^{2}$ Institut für Theoretische Physik III, Universität Bayreuth, 95440 Bayreuth, Germany \\ ${ }^{3}$ Hitachi Cambridge Laboratory, Hitachi Europe Ltd., Cambridge CB3 OHE, United Kingdom
}

(Received 2 September 2014; published 30 March 2015)

\begin{abstract}
We demonstrate a new method to realize the population inversion of a single InGaAs/GaAs quantum dot excited by a laser pulse tuned within the neutral exciton phonon sideband. In contrast to the conventional method of inverting a two-level system by performing coherent Rabi oscillation, the inversion is achieved by rapid thermalization of the optically dressed states via incoherent phonon-assisted relaxation. A maximum exciton population of $0.67 \pm 0.06$ is measured for a laser tuned $0.83 \mathrm{meV}$ to higher energy. Furthermore, the phonon sideband is mapped using a two-color pump-probe technique, with its spectral form and magnitude in very good agreement with the result of path-integral calculations.
\end{abstract}

DOI: 10.1103/PhysRevLett.114.137401

PACS numbers: 78.67.Hc, 03.67.Lx, 42.50.Hz, 78.45.+h

It is a basic tenet of laser physics that a population inversion cannot be achieved through incoherent excitation of a two-level atom. At best, a laser pulse with duration much longer than the coherence time of the two-level system $T_{2}$ can only drive the system to the transparency point where the populations of the upper and lower levels are equal [1]. However, if the two-level atom is coupled to a vibrational continuum, it has been predicted that inversion can be possible even in the incoherent regime through the interaction of the dressed states with the Boson bath [2]. Excitons in semiconductor quantum dots (QDs) form a near ideal system for investigating these effects, since their behavior approximates well to that of a two-level atom [3], while their coupling to the acoustic phonons in the crystal provides a mechanism to thermalize the dressed states.

The possibility of creating population inversion in QDs through phonon coupling was first investigated for microwave-driven electrostatic quantum dots [4]. Recently, it has been demonstrated that the conditions for population inversion can be met via a microwave Raman effect $[5,6]$. Theoretical work has indicated that similar effects should be possible for optically driven excitons [7-11]. The underlying mechanism is the coupling of the excitons to longitudinal acoustic (LA) phonons through the deformation potential [12], which generates sidebands in the excitonic spectra [13] that can also be observed in fourwave mixing [14] and resonance fluorescence experiments [15], as well as through off-resonant coupling of excitons to nanocavities [16,17]. In a strong driving field regime,

Published by the American Physical Society under the terms of the Creative Commons Attribution 3.0 License. Further distribution of this work must maintain attribution to the author(s) and the published article's title, journal citation, and DOI. evidence for phonon-induced relaxation between optically dressed states is observed in the intensity damping of Rabi rotations [18-20], and more recently in adiabatic rapid passage experiments [21,22].

In this Letter we report a population inversion of the excitonic two-level system of a single InGaAs/GaAs QD excited by a strong laser pulse tuned into the phonon sideband of the neutral exciton transition following the theoretical proposal of Ref. [9]. The population inversion is achieved in an incoherent regime where the dephasing time is shorter than the laser pulse duration. Pump-probe measurements are presented, where the phonon-assisted population inversion is observed clearly as a gainlike dip in the photocurrent absorption spectrum. Furthermore, the dependence of the exciton population on the pump detuning is measured. The experiments are supplemented by simulations based on the path-integral approach described in Ref. [23] and good agreement is obtained with the theory. This work may form the basis of quasiresonantly pumped single QD lasers. It also shows that the phonon-assisted quasiresonant excitation scheme can be used to create spinpolarized excitons whilst allowing the QD emission to be spectrally filtered from the pump laser, with potential applications for QD-based high-brightness single photon sources [17] and on-chip quantum optical circuits [24].

The physical mechanism for generating the population inversion can be understood as follows. A circularly polarized laser pulse with a large pulse area excites the neutral exciton transition of an InGaAs/GaAs quantum dot at a positive detuning within the phonon sideband, which typically peaks around $1 \mathrm{meV}$ above the exciton [see Fig. 4(a) inset]. Since the laser bandwidth of $0.2 \mathrm{meV}$ is large compared with the fine-structure splitting of $13 \mu \mathrm{eV}$, the dynamics of the exciton spin can be neglected [25]. Also, since the laser is far detuned from the two-photon 
biexciton transition, the QD can be treated as a two-level system composed of two bare states: a crystal ground state $|0\rangle$ and neutral exciton $|X\rangle$ [see Fig. 1(a)(i)], with respective populations $C_{0}$ and $C_{X}$. With the presence of a laser pulse, the two bare states are optically dressed [see Figs. 1(a)(ii) and 1(a)(iii)]. In the rotating frame, $\left|0_{R}\right\rangle$ comprises the crystal ground state and the incoming laser photons. $\left|X_{R}\right\rangle$ comprises the exciton state and laser field with one photon less. The Hamiltonian in a rotating frame reads

$$
H_{\mathrm{QD}}=-\hbar \Delta\left|X_{R}\right\rangle\left\langle X_{R}\left|+\frac{\hbar \Omega(t)}{2}\right| 0_{R}\right\rangle\left\langle X_{R}\right|+\text { H.c., }
$$

where the detuning $\Delta=\omega_{L}-\omega_{X}$, and $\Omega(t)$ is the Rabi frequency, which varies in time following the envelope of the laser pulse. The energy eigenstates of $H_{\mathrm{QD}},|\alpha\rangle,|\beta\rangle$ are described by an admixing angle $2 \theta(t)=\arctan (\Omega(t) / \Delta)$, and are split by the effective Rabi energy $\hbar \Lambda(t)=$ $\hbar \sqrt{\Delta^{2}+\Omega(t)^{2}}$. We define the bare pulse area $\Theta=$ $\int_{-\infty}^{+\infty} \Omega(t) d t$.

The QD resides in a crystal lattice, and the exciton interacts with the LA phonons due to the deformation potential [12]. In the absence of a laser field, the excitonphonon interaction leads to nonexponential pure dephasing of the excitonic dipole, as observed in time-resolved

(a)
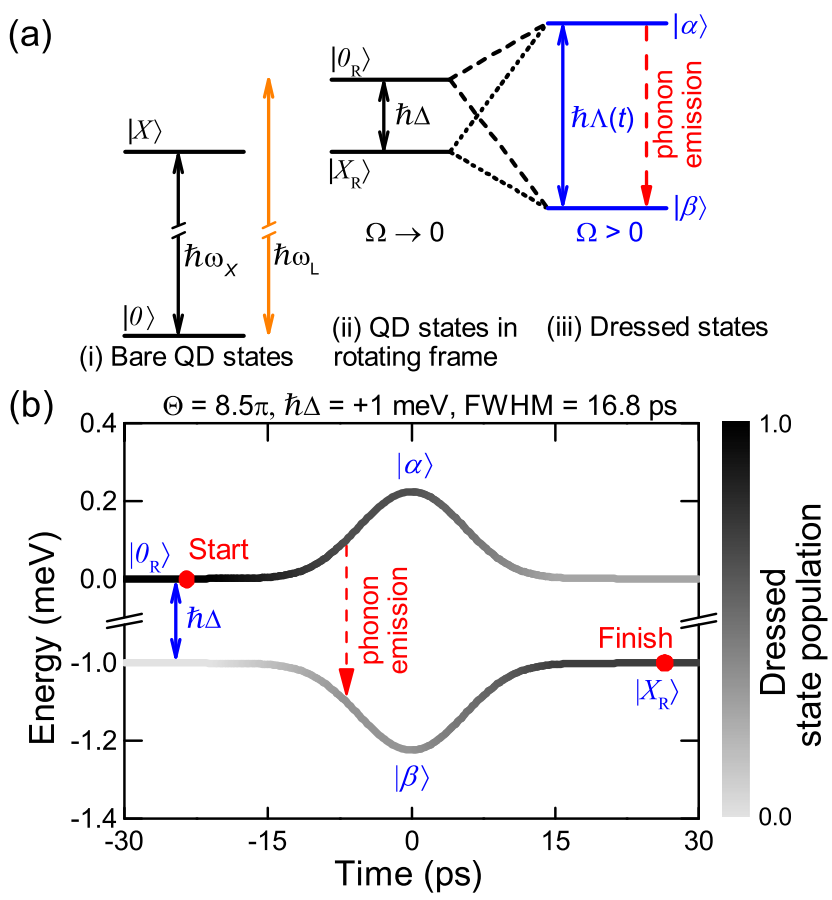

FIG. 1 (color online). (a) Bare QD states viewed in the (i) lab frame and (ii) rotating frame. $|0\rangle,|X\rangle$ and $\left|0_{R}\right\rangle,\left|X_{R}\right\rangle$ denote the ground state and exciton state in the lab frame and rotating frame, respectively. $\hbar \omega_{X}$ is the exciton transition energy. $\omega_{L}$ is the angular frequency of the laser. $\hbar \Delta$ is the positive detuning from the exciton transition. (iii) Optically dressed states $|\alpha\rangle$ and $|\beta\rangle$. (b) Evolution of the dressed QD energy levels with time during the absorption of an $8.5 \pi$ pulse with $\hbar \Delta=+1 \mathrm{meV}$. The gray scale of the curves corresponds to the instantaneous population of each state. four-wave mixing experiments [14]. In the presence of a strong laser, both dressed states have an excitonic component, thereby enabling relaxation between them by the emission of a phonon with energy equal to the effective Rabi splitting $\hbar \Lambda$, as shown in Fig. 1(a)(iii) for the case of positive detuning. This process gives rise to intensity damping of Rabi rotations when resonantly pumping [18,19], and also explains the difference in the population inversions created by adiabatic rapid passage when using laser pulses of positive and negative chirp [21,22].

Figure 1(b) depicts the dynamics in the rotating frame of the phonon-assisted population inversion of the quantum dot exciton. For a positively detuned laser $(\Delta>0)$, the state $\left|0_{R}\right\rangle$ initially coincides with the higher-energy dressed state $|\alpha\rangle$. When the laser is applied, excitonic admixture to the state $|\alpha\rangle$ occurs, activating relaxation to the lower-energy dressed state $|\beta\rangle$ by phonon emission. In the lab frame [see Fig. 1(a)(i)], this process corresponds to the absorption of a photon together with the creation of an exciton and the emission of a phonon. The gray scale indicates the time-dependent populations of each dressed state, as calculated by our pathintegral method, and shows the continuous transfer of population from $|\alpha\rangle$ to $|\beta\rangle$. As the laser intensity drops off at the end of the pulse, the admixing of the dressed states is reduced and the phonon relaxation is deactivated. If the time-integrated phonon relaxation is strong, the final occupation of the lower-energy, excitonlike, dressed state can dominate and a population inversion in the excitonic basis occurs. According to our theoretical model, near-unity exciton population that is robust against variations in both the pulse area and the detuning can be achieved at high pulse areas [9]. The amount of inversion is ultimately limited by the thermal occupation of the two states to $C_{X}-C_{0}=\tanh \left(\hbar \Delta / 2 k_{B} T\right)$ [9]. In our experiments with $\hbar \Delta=0.83 \mathrm{meV}$ and $T=4.2 \mathrm{~K}$, this implies a maximum exciton population of 0.91 .

The experiments were performed on a device consisting of a layer of InGaAs/GaAs quantum dots embedded in the intrinsic region of an $n$-i-Schottky diode structure. The sample is held at $4.2 \mathrm{~K}$ in a helium bath cryostat and is excited at normal incidence with circularly polarized Gaussian pulses, derived by pulse shaping of the output from a 100 fs Ti:sapphire laser with a repetition rate of 76.2 MHz. The FWHM of the electric field amplitude is 16.8 ps. Single QD peaks are observed in our sample at energies close to $1.3 \mathrm{eV}$. Photocurrent detection is used, where photogenerated electron-hole pairs tunnel from the quantum dot under an applied electric field. The amplitude of the photocurrent signal reflects the final occupation of the exciton or biexciton states. Full details of the wafer structure can be found in Ref. [26].

To demonstrate the phonon-assisted population inversion, two-pulse pump probe experiments were performed. First, the zero-phonon neutral exciton transition was found by measuring the absorption spectrum of a single $\pi$ pulse (blue line in Fig. 2). Then, the pump pulse was detuned by $+0.83 \mathrm{meV}$, where the exciton population is predicted to be 
most efficiently created [see blue line in Fig. 4(a)], and the probe energy was scanned through the transition. The probe pulse area is $\pi$ and the pump pulse area is $8.46 \pi$, the maximum available in our setup. The delay time between the pump and probe pulses $\tau_{\text {delay }}$ ranges from 10 to 34 ps [27].

As illustrated in the energy-level diagram shown in Fig. 2, the photon energy of the probe pulse and its polarization relative to the pump selects the transition that is probed. We consider first the case for a copolarized probe pulse, where both the pump and probe pulses have the same $\sigma^{+}$polarization. In this case, the pump pulse (orange arrow) excites a dephased, mixed exciton population $C_{X}$ (see the inset in Fig. 2). Since the probe has a pulse area of $\pi$, it exchanges the $|0\rangle$ population with the $|X\rangle$ population when it is resonant with the neutral exciton transition (see black arrow in the inset to Fig. 2), resulting in a change in photocurrent proportional to the populations after the pump but before the probe: $\Delta \mathrm{PC}_{0-X} \propto C_{0}-C_{X}$ [27]. $\triangle \mathrm{PC}$ is the change in the photocurrent signal resulting from the dot that is induced by the probe pulse. $\triangle \mathrm{PC}$ is measured relative to the photocurrent measured for a detuned probe. A key signature of population inversion is that $\triangle \mathrm{PC}_{0-X}$ should be negative, independent of the photocurrent to exciton population calibration. Figure 2 shows the experimental results (black). The dip at zero detuning clearly demonstrates that a population inversion has been achieved between the $|0\rangle$ and $|X\rangle$ states.

The red line in Fig. 2 shows the results obtained for crosspolarized excitation ( $\sigma^{+}$pump, $\sigma^{-}$probe). At zero detuning, the probe addresses the orthogonally polarized

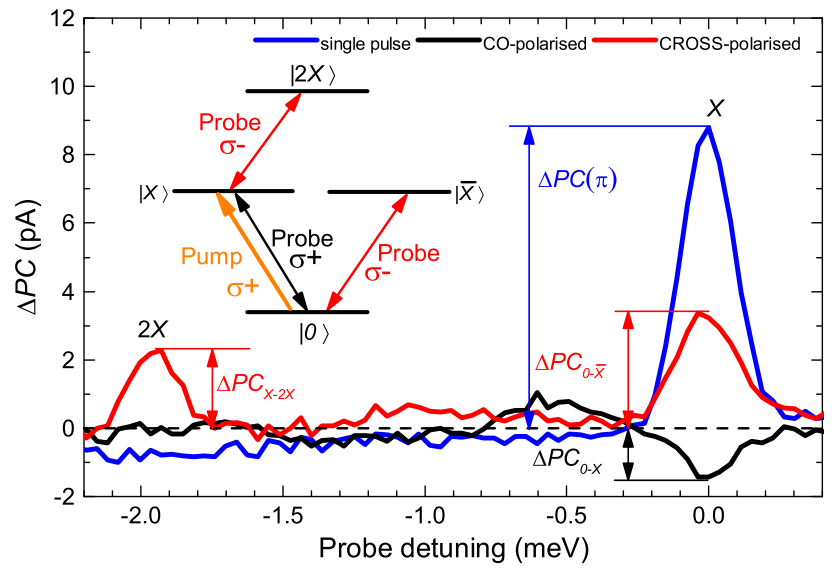

FIG. 2 (color online). Photocurrent signal $\triangle \mathrm{PC}$ as a function of the probe detuning. Blue: a single probe-pulse only spectrum is presented for reference. Two pulse spectra where the probe is copolarized (black) and crosspolarized (red) with the pump. Pump detuning $=+0.83 \mathrm{meV}$ and $\tau_{\text {delay }}=10 \mathrm{ps}$. The peak at a detuning of $-1.96 \mathrm{meV}$ corresponds to the $|X\rangle \rightarrow|2 X\rangle$ transition. Inset: energy level diagram for the exciton-biexciton system. Arrows represent transitions induced by the $\sigma^{+}$polarized pump pulse tuned to the phonon sideband and the $\sigma^{+}\left(\sigma^{-}\right)$polarized probe pulse tuned either to the exciton or biexciton transition. $X$ and $\bar{X}$ label the orthogonally circularly polarized exciton states. $2 X$ denotes the biexciton state. exciton transition $|0\rangle \rightarrow|\bar{X}\rangle$ as shown by the lower red arrow in the inset to Fig. 2, providing a measure of the occupation of the crystal ground state $C_{0}$. The amplitude of the peak at zero detuning falls to less than half the amplitude measured by the single pulse, again confirming that a population inversion between $|0\rangle$ and $|X\rangle$ has been achieved since $\Delta \mathrm{PC}_{0-\bar{X}} \propto C_{0}-C_{\bar{X}}$. The second peak at a detuning of $-1.96 \mathrm{meV}$ corresponds to the $|X\rangle \rightarrow|2 X\rangle$ transition, and provides a third measure of $C_{X}$ (see Ref. [27]). The absence of the biexciton peak in the copolarized spectra proves that the phonon-assisted channel is spin preserving because the phonon-assisted relaxation (few picoseconds) [30] is much faster than exciton spin relaxation (few nanoseconds) [31].

Figure 3 plots the occupation of the neutral exciton versus the area of the pump pulse. The three data points for each pulse area are obtained from an analysis of the co- and crosspolarized signals at zero detuning, and from the biexciton peak. The exciton population is deduced by comparison with the neutral exciton photocurrent peak for resonant excitation with a single $\pi$ pulse (for details see Ref. [27]). Since the electron can tunnel out from the QD before the arrival of the probe, which reduces the measured exciton population created by the pump, a correction is made to account for an electron tunneling time of $\sim 50 \mathrm{ps}$ [27]. The three measurements of the exciton population are in close agreement, and pass the transparency point, $C_{X}=0.5$, at a pulse area of $6.5 \pi-7 \pi$. The largest exciton population observed is $0.67 \pm 0.06$ for a pump pulse detuned by $+0.83 \mathrm{meV}$, limited by the power available in our setup. The blue line in Fig. 3 shows the results of our path-integral simulations, which quantitatively reproduce the experiments.

To investigate the dependence of the phonon-assisted population inversion on the pump frequency, a series of two-color photocurrent spectra, similar to Fig. 2, were measured for a crosspolarized $7.24 \pi$ pump pulse as a function of pump detuning with a $\pi$ probe. Figure 4(a) presents the exciton population generated by the pump pulse.

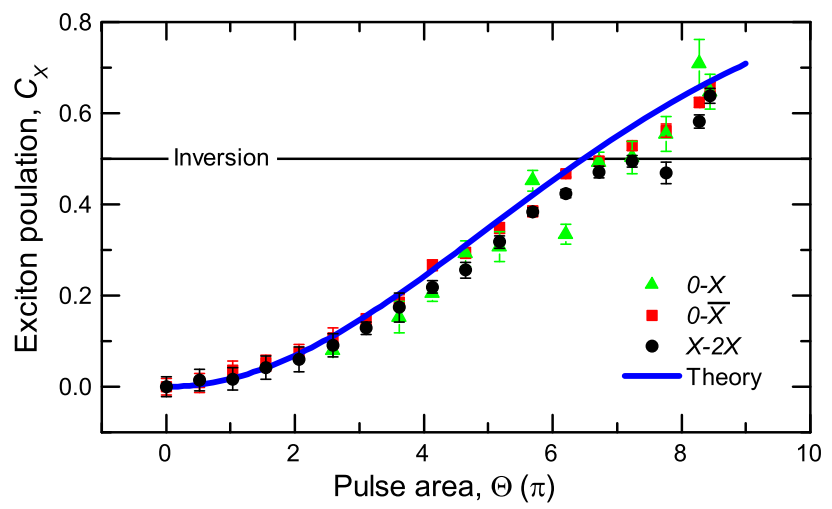

FIG. 3 (color online). The exciton population $C_{X}$ generated by the pump pulse with $\hbar \Delta=+0.83 \mathrm{meV}$, as extracted from the exciton and biexciton peak in two-pulse spectra versus the pump pulse area. For a full derivation, see Ref. [27]. Blue: calculated $C_{X}$. 

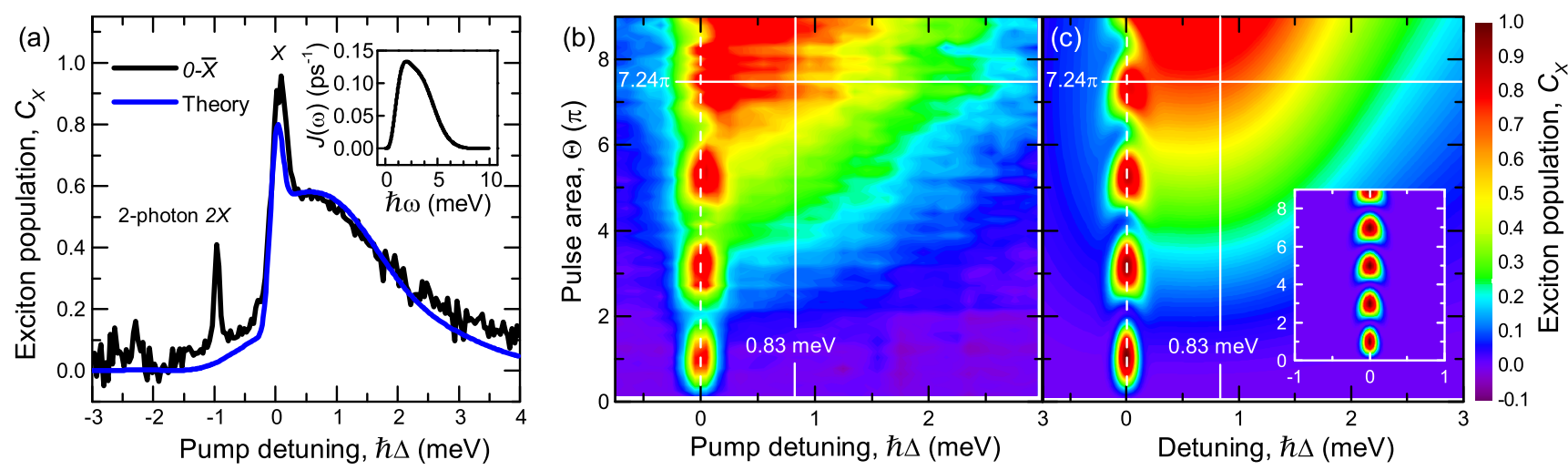

FIG. 4 (color online). (a) Exciton population created by a $7.24 \pi$ pump as a function of the pump detuning. $\tau_{\text {delay }}=15$ ps. Blue: calculated $C_{X}$. Inset: theoretical spectral density of the exciton-phonon interaction $J(\omega)$. (b) Experimentally obtained $C_{X}$ versus $\Theta$ and $\hbar \Delta$ with $\tau_{\text {delay }}=33.6$ ps. (c) Path-integral results for the same pulse area and detuning range as in (b). The vertical line is where $\hbar \Delta=+0.83 \mathrm{meV}$ and the horizontal line is for $\Theta=7.24 \pi$ as shown in Figs. 3 and 4(a), respectively. Inset: calculated values with no phonon interaction. All plots in (b) and (c) use the same color scale.

The spectrum has three features. At zero detuning there is a pulse width-limited peak corresponding to the zero-phonon $|0\rangle \rightarrow|X\rangle$ transition. At positive detuning there is a broad feature due to phonon emission. The absence of discrete features in the phonon sideband indicates that phonon confinement effects are weak, as expected given the similar lattice properties of the QD and barrier materials. In principle, there can also be a phonon feature at negative detuning due to phonon absorption. However, the phonon absorption is negligible at low temperature. The third feature is a narrow peak at a pump detuning of $-1 \mathrm{meV}$, corresponding to the two-photon $|0\rangle \rightarrow|2 X\rangle$ biexciton transition [32], indicating that the pump pulse is slightly elliptically polarized.

In the theory, the phonon influence on the dot dynamics is mediated mainly by the phonon spectral density: $J(\omega)=$ $\sum_{\mathbf{q}}\left|\gamma_{\mathbf{q}}\right|^{2} \delta\left(\omega-\omega_{\mathbf{q}}\right)$, where $\gamma_{\mathbf{q}}$ is the exciton-phonon coupling. In the absence of detailed information on the shape of the QD we consider spherically symmetric, parabolic potentials. For bulk LA phonons coupled via the deformation potential we then obtain [23]

$$
J(\omega)=\frac{\omega^{3}}{4 \pi^{2} \rho \hbar v_{c}^{5}}\left[D_{e} e^{\left(-\omega^{2} a_{e}^{2} / 4 v_{c}^{2}\right)}-D_{h} e^{\left(-\omega^{2} a_{h}^{2} / 4 v_{c}^{2}\right)}\right]^{2},
$$

where $\rho$ is the mass density, $v_{c}$ is the sound velocity, and $D_{e(h)}$ denote the deformation potential constants for electrons(holes). These material parameters are taken from the literature and are given explicitly in the Supplemental Material [27], while the electron(hole) confinement lengths $a_{e(h)}$ are used as fitting parameters. Good agreement with our experiments is obtained for $a_{e}=4.5 \mathrm{~nm}$ and $a_{h}=1.8 \mathrm{~nm}$, resulting in the spectral density shown in the inset of Fig. 4(a). We note that the low frequency asymptote $\sim \omega^{3}$ is characteristic for bulk acoustic phonons and occurs independent of the material or the dot shape. On the other hand, the Gaussians in Eq. (2) result from the Fourier transforms of the electron and hole probability densities, reflecting the assumption of parabolic confinement potentials.

The blue line in Fig. 4(a) shows the theoretical values of the exciton population generated by the pump pulse, which excellently replicates the broadband feature observed at positive detuning. The line shape of this feature is implicitly determined by $J(\omega)$; more detailed information on $J(\omega)$ can be obtained here than from fitting the intensity damping of Rabi rotations [19]. Equation (2) shows that the high frequency behavior of $J(\omega)$ follows the Fourier transform of the electron and hole probability densities, and so a line shape analysis in principle provides a way to obtain insight on the spatial distributions of the electrons and holes. However, accessing this information would require more detailed studies, which are beyond the scope of the present Letter. The asymmetry of the spectrum with respect to the sign of $\hbar \Delta$ unambiguously proves that the population created by the off-resonant pump pulse is the result of phonon-assisted relaxation into the lower energy dressed state [33]. This is further demonstrated by a calculation without exciton-phonon coupling, where no broad sideband is observed [see inset in Fig. 4(c)]. We also note that the measured low-energy phonon sideband is stronger than expected. This may be due to an elevated temperature of about 6-7 K caused by heating of the sample by the laser.

Figures 4(b) and 4(c) compare the exciton population generated by the pump pulse measured as in Fig. 4(a) at different pulse areas with corresponding path-integral calculations. On resonance, the zero-phonon line exhibits intensity-damped Rabi rotations. To positive detuning, there is the phonon-emission sideband that broadens for higher pulse areas. The calculations are in good agreement with the experimental data, further confirming the model. Even the slight shift of the resonant peaks towards higher energies with increasing pulse area is reproduced by the theory.

In conclusion, we have experimentally demonstrated the population inversion of a neutral exciton in a single QD excited by a quasiresonant laser pulse tuned within the 
exciton phonon sideband. The population inversion arises due to the incoherent phonon-induced relaxation between optically dressed states that arises at high driving intensities. Phonon interactions, which are usually a hindrance in quantum dot physics, in this case enable the population inversion and lead to qualitatively distinct behavior. The phonon-mediated population inversion we report may have potential as the basis of quasiresonantly pumped single QD lasers with dots embedded in cavities, and as high repetition rate single photon sources [17], with easy spectral filtering of excitation lasers, especially relevant to on-chip geometries [24].

This work was funded by the EPSRC (UK) EP/J007544/1. M. G., A.M.B., and V.M. A. gratefully acknowledge the financial support from Deutsche Forschungsgemeinschaft via the Project No. AX 17/7-1. The authors thank H. Y. Liu and M. Hopkinson for sample growth.

Note added in proof.-Following submission of our paper, we became aware of related results by two other groups $[35,36]$.

*fengliu@sheffield.ac.uk

[1] A. E. Siegman, Lasers (University Science Books, Oxford, 1986).

[2] A. J. Leggett, S. Chakravarty, A. T. Dorsey, M. P. A. Fisher, A. Garg, and W. Zwerger, Rev. Mod. Phys. 59, 1 (1987).

[3] T. H. Stievater, X. Li, D. G. Steel, D. Gammon, D. S. Katzer, D. Park, C. Piermarocchi, and L. J. Sham, Phys. Rev. Lett. 87, 133603 (2001)

[4] J. R. Petta, A. C. Johnson, C. M. Marcus, M. P. Hanson, and A. C. Gossard, Phys. Rev. Lett. 93, 186802 (2004).

[5] T. M. Stace, A. C. Doherty, and S. D. Barrett, Phys. Rev. Lett. 95, 106801 (2005).

[6] J. I. Colless, X. G. Croot, T. M. Stace, A. C. Doherty, S. D. Barrett, H. Lu, A.C. Gossard, and D. J. Reilly, Nat. Commun. 5, 3716 (2014).

[7] M. Glässl, A. Vagov, S. Lüker, D. E. Reiter, M. D. Croitoru, P. Machnikowski, V. M. Axt, and T. Kuhn, Phys. Rev. B 84, 195311 (2011).

[8] D. E. Reiter, S. Lüker, K. Gawarecki, A. Grodecka-Grad, P. Machnikowski, V. M. Axt, and T. Kuhn, Acta Phys. Pol. A 122, 1065 (2012).

[9] M. Glässl, A. M. Barth, and V. M. Axt, Phys. Rev. Lett. 110, 147401 (2013).

[10] S. Hughes and H. J. Carmichael, New J. Phys. 15, 053039 (2013).

[11] D. E. Reiter, T. Kuhn, M. Glässl, and V. M. Axt, J. Phys. Condens. Matter 26, 423203 (2014).

[12] B. Krummheuer, V. M. Axt, and T. Kuhn, Phys. Rev. B 65, 195313 (2002).

[13] L. Besombes, K. Kheng, L. Marsal, and H. Mariette, Phys. Rev. B 63, 155307 (2001).

[14] P. Borri, W. Langbein, U. Woggon, V. Stavarache, D. Reuter, and A. D. Wieck, Phys. Rev. B 71, 115328 (2005).
[15] S. Weiler, A. Ulhaq, S. M. Ulrich, D. Richter, M. Jetter, P. Michler, C. Roy, and S. Hughes, Phys. Rev. B 86, 241304 (2012).

[16] U. Hohenester, A. Laucht, M. Kaniber, N. Hauke, A. Neumann, A. Mohtashami, M. Seliger, M. Bichler, and J. J. Finley, Phys. Rev. B 80, 201311 (2009).

[17] K. H. Madsen, S. Ates, J. Liu, A. Javadi, S. M. Albrecht, I. Yeo, S. Stobbe, and P. Lodahl, Phys. Rev. B 90, 155303 (2014).

[18] A. J. Ramsay, A. V. Gopal, E. M. Gauger, A. Nazir, B. W. Lovett, A. M. Fox, and M. S. Skolnick, Phys. Rev. Lett. 104, 017402 (2010).

[19] A. J. Ramsay, T. M. Godden, S. J. Boyle, E. M. Gauger, A. Nazir, B. W. Lovett, A. M. Fox, and M. S. Skolnick, Phys. Rev. Lett. 105, 177402 (2010).

[20] L. Monniello, C. Tonin, R. Hostein, A. Lemaitre, A. Martinez, V. Voliotis, and R. Grousson, Phys. Rev. Lett. 111, 026403 (2013).

[21] Y.-J. Wei, Y.-M. He, M.-C. Chen, Y.-N. Hu, Y. He, D. Wu, C. Schneider, M. Kamp, S. Höfling, C.-Y. Lu et al., Nano Lett. 14, 6515 (2014).

[22] R. Mathew, E. Dilcher, A. Gamouras, A. Ramachandran, H. Y. Shi Yang, S. Freisem, D. Deppe, and K. C. Hall, Phys. Rev. B 90, 035316 (2014).

[23] A. Vagov, M. D. Croitoru, M. Glässl, V. M. Axt, and T. Kuhn, Phys. Rev. B 83, 094303 (2011).

[24] M. Makhonin, J. Dixon, R. Coles, B. Royall, I. J. Luxmoore, E. Clarke, M. Hugues, M. Skolnick, and A. M. Fox, Nano Lett. 14, 6997 (2014).

[25] Q. Q. Wang, A. Muller, M. T. Cheng, H. J. Zhou, P. Bianucci, and C. K. Shih, Phys. Rev. Lett. 95, 187404 (2005).

[26] R. S. Kolodka, A. J. Ramsay, J. Skiba-Szymanska, P. W. Fry, H. Y. Liu, A. M. Fox, and M. S. Skolnick, Phys. Rev. B 75, 193306 (2007).

[27] See Supplemental Material at http://link.aps.org/ supplemental/10.1103/PhysRevLett.114.137401 for detailed derivation of the exciton population and description of the model, which includes Refs. [9,23,26,28,29].

[28] S. J. Boyle, A. J. Ramsay, F. Bello, H. Y. Liu, M. Hopkinson, A. M. Fox, and M. S. Skolnick, Phys. Rev. B 78, 075301 (2008).

[29] B. Krummheuer, V. M. Axt, T. Kuhn, I. D'Amico, and F. Rossi, Phys. Rev. B 71, 235329 (2005).

[30] D. P. S. McCutcheon and A. Nazir, New J. Phys. 12, 113042 (2010).

[31] K. Roszak, V. M. Axt, T. Kuhn, and P. Machnikowski, Phys. Rev. B 76, 195324 (2007).

[32] S. Stufler, P. Machnikowski, P. Ester, M. Bichler, V. M. Axt, T. Kuhn, and A. Zrenner, Phys. Rev. B 73, 125304 (2006).

[33] We note that in single pulse experiments at $15 \mathrm{~K}$, more symmetric phonon sidebands can be observed [34].

[34] A. J. Ramsay, T. M. Godden, S. J. Boyle, E. M. Gauger, A. Nazir, B. W. Lovett, A. V. Gopal, A. M. Fox, and M. S. Skolnick, J. Appl. Phys. 109, 102415 (2011).

[35] P.-L. Ardelt, L. Hanschke, K. A. Fischer, K. Müller, A. Kleinkauf, M. Koller, A. Bechtold, T. Simmet, J. Wierzbowski, H. Riedl, G. Abstreiter, and J. J. Finley, Phys. Rev. B 90, 241404 (2014).

[36] S. Bounouar, M. Müller, A. M. Barth, M. Glässl, V. M. Axt, and P. Michler, arXiv:1408.7027. 